\title{
Adaptabilidade e estabilidade de cultivares de alfafa em relação a diferentes épocas de corte
}

\author{
Adaptability and stability of alfalfa cultivars in relation to different yielding dates \\ Reinaldo de Paula Ferreira ${ }^{1}$ Milton de Andrade Botrel ${ }^{2}$ Ana Claúdia Ruggieri ${ }^{3}$ \\ Antônio Vander Pereira ${ }^{1}$ Antônio Daniel Fernandes Coelho ${ }^{4}$ \\ Francisco José da Silva Lédo ${ }^{1}$ Cosme Damião Cruz ${ }^{5}$
}

\section{RESUMO}

O experimento foi conduzido na Estação Experimental de Zootecnia de Sertãozinho-SP. O delineamento experimental foi o de blocos casualizados com 3 repetições, sendo a parcela constituída de 5 fileiras de $5 \mathrm{~m}$ de comprimento, espaçados 0,2 m. Avaliou-se a produção de matéria seca de 35 cultivares de alfafa em 29 cortes realizados no período de outubro/1996 a março/1999. O estudo da adaptabilidade e estabilidade das cultivares ao longo dos 29 cortes, foi realizado por meio das metodologias de Eberhart e Russell e Lin e Binns. Verificou-se a existência de interação cultivares $x$ cortes, demonstrando que o comportamento relativo das cultivares não era o mesmo nos diferentes cortes. Das 35 cultivares avaliadas, 14 apresentaram adaptabilidade geral $\left(\beta_{1 i}=1\right) e$ boa previsibilidade $\left(\sigma^{2} d i=0\right)$, segundo a metodologia de "Eberhart e Russell". Das 10 cultivares que apresentaram maior adaptabilidade e estabilidade (menores $P$ ), segundo a metodologia de Lin e Binns sete também apresentaram adaptabilidade geral e boa previsibilidade segundo a metodologia de Eberhart e Russell. As cultivares SW 8210, Victoria SP, MH 15, 5888, Araucana, BR 1 e BR 3 foram as que apresentaram maior adaptabilidade e estabilidade de comportamento, pelos dois métodos utilizados.

Palavras-chave: interação cultivar x ambiente, épocas de corte, Medicago sativa $L$.

\section{ABSTRACT}

An experiment was carried out at the Animal Science Experimental Station of Sertãozinho, São Paulo, Brazil. The experimental design was a randomized block design, with three replications. Plots were constituted of 5 rows, with $5 \mathrm{~m}$ long, separated by $0.2 \mathrm{~m}$. Thirty-five alfalfa cultivars were evaluated for dry matter yield in 29 yielding dates, from October, 1996 to March, 1999. Adaptability and stability study was stimated with "Eberhart \& Russell" and "Lin \& Binns" methods. Significant cultivar $x$ yielding dates interaction was verified, indicating that the relative behavior of the cultivars was not the same in the different yielding dates. Among the 35 evaluated cultivars, 14 presented wide adaptability $\left(\beta_{1 i}=1\right)$ and good predicability $\left(\sigma^{2} d i=0\right)$, according to the "Eberhart \& Russell" method. Among 10 cultivars that had high adaptability and predicability (smallest values of $P$ ), estimated by "Lin \& Binns" method, seven also had high aidaptability and good predicability, estimated by "Eberhart \& Russell" method. The cultivars SW 8210, Victoria SP, MH 15, 5888, Araucana, BR 1 and BR 3 had the high test adaptability and predicability, estimations obtained in both employed methods.

Key words: cultivar $x$ environment interaction, yielding dates, Medicago sativa $L$.

\section{INTRODUÇÃO}

A alfafa (Medicago sativa L.) é uma planta perene, originária da Ásia, de onde se difundiu para a Europa e as Américas (MONTEIRO et al., 1999). No Brasil, foi introduzida no Rio Grande do Sul por imigrantes europeus e depois foi levada para os Estados de Santa Catarina, Paraná e São Paulo.

Atualmente, tem havido um crescente interesse por esta leguminosa. Segundo VILELA (1992), o aumento da área plantada no país e sua expansão para a Região Sudeste devem-se à crescente

${ }^{1}$ Engenheiro. Agrônomo, Doutor, Embrapa Gado de Leite. Rua Eugênio do Nascimento, 610, 36038-330, Juiz de Fora, MG.

${ }^{2}$ Engenheiro Agrônomo, Mestre, Embrapa Gado de Leite.

${ }^{3}$ Engenheiro Agrônomo, Instituto de Zootecnia, Nova Odessa, SP.

${ }^{4}$ Engenheiro Agrônomo, Bolsista recém-doutor, Embrapa Gado de Leite.

${ }^{5}$ Engenheiro Agrônomo, Doutor, Professor Titular, Departamento Biologia Geral, Universidade Fedederal de Viçosa, 36571-000,

Viçosa, MG. 
implantação de sistemas intensivos de produção leiteira, o que tem aumentado a demanda de alimentos de alto valor nutritivo.

Com o aumento da importância dessa forrageira no Brasil, torna-se necessária a seleção de cultivares mais adaptadas às nossas condições edafoclimáticas, de maneira a permitir maiores rendimentos e redução dos custos com a cultura. Uma das grandes dificuldades dos melhoristas diz respeito à interação genótipos x ambientes, caracterizada pelo comportamento dos genótipos que não mantêm o mesmo desempenho relativo nos diversos ambientes onde são cultivados. Quando as interações são do tipo complexo, uma cultivar superior num ambiente poderá apresentar desempenho inferior em outro ambiente. Na presença de interações, recomenda-se a estratificação ambiental ou o uso de cultivares de ampla adaptabilidade e estabilidade (CRUZ \& REGAZZI, 1997). Estudos sobre adaptabilidade e estabilidade têm sido empregados no melhoramento de plantas em diversas culturas, como no algodão, arroz e milho (CARVALHO et al., 1995; SILVA et al., 1995; OLIVEIRA et al., 1999).

Nas plantas forrageiras, uma fonte extra de variação ambiental é dada pelos diferentes cortes realizados ao longo do ano, quando as plantas são submetidas a diferentes estações climáticas. Condições ambientais tais como temperatura, umidade relativa, fotoperíodo e precipitação variam muito ao longo do ano, podendo contribuir para a interação genótipos $\mathrm{x}$ cortes. O objetivo deste trabalho foi avaliar a adaptabilidade e a estabilidade da produção de matéria seca de 35 cultivares de alfafa, submetidas a 29 cortes.

\section{MATERIAL E MÉTODOS}

O experimento foi realizado na Estação Experimental de Zootecnia de Sertãozinho, SP, localizada a $21^{\circ} 08^{\prime}$ de latitude Sul e $47^{0}$ de longitude Oeste. Utilizou-se o equivalente a $20 \mathrm{~kg} / \mathrm{ha}$ de sementes puras viáveis, inoculadas com Rhizobium melilotii e, em seguida, peletizadas com calcário dolomítico. A semeadura foi realizada em sulcos de $2 \mathrm{~cm}$ de profundidade, em 11/04/96.

$\mathrm{O}$ ensaio foi instalado no delineamento experimental de blocos casualizados, com três repetições. Cada parcela foi constituída de cinco fileiras de $5 \mathrm{~m}$ de comprimento, com $0,2 \mathrm{~m}$ entre fileiras. Foi considerado como área útil as três fileiras centrais, eliminando-se $0,5 \mathrm{~m}$ de cada extremidade. Utilizaram-se 35 cultivares de alfafa, de diversas procedências, nos quais foi avaliada a produção de matéria seca de cada cultivar. Os cortes foram feitos a $5 \mathrm{~cm}$ acima do solo e ocorriam sempre que $50 \%$ das cultivares atingiam $10 \%$ de floração. Procedeu-se a irrigação por aspersão, sempre que necessário. Foram realizados 29 cortes no período de outubro de 1996 a março de 1999.

A análise de variância foi realizada considerando os tratamentos no esquema de parcela subdividida no tempo, sendo as parcelas representadas pelas cultivares e as subparcelas pelos cortes. Foi utilizado o seguinte modelo (RAMALHO et al., 2000):

$$
\mathrm{Y}_{\mathrm{ijk}}=\mu+\mathrm{B}_{\mathrm{j}}+\mathrm{P}+\varepsilon_{\mathrm{ij}}+\mathrm{S}_{\mathrm{k}}+\theta_{\mathrm{jk}}+\mathrm{PS}_{\mathrm{ik}}+\delta_{\mathrm{ijk}}
$$

em que $Y_{\mathrm{ijk}}$ : valilor da produção de matéria seca da cultivar $\mathrm{i}(\mathrm{i}=1,2 \ldots 35)$, no corte $\mathrm{j}(\mathrm{j}=1,2 . .29)$ e na repetição $\mathrm{k}(\mathrm{k}=1,2$ e 3$) ; \mu$ : média geral; $\mathrm{B}_{\mathrm{j}}$ : efeito do bloco j; $\mathrm{P}_{\mathrm{i}}$ : efeito da cultivar $\mathrm{i} ; \varepsilon_{\mathrm{i}}$ : erro aleatório a; $\mathrm{S}_{\mathrm{k}}$ : efeito do corte $\mathrm{k} ; \theta_{\mathrm{jk}}$ : erro aleatốrio $\mathrm{b} ; \mathrm{PS}_{\mathrm{ik}}$ : efeito da interação da cultivar i com o corte $\mathrm{k}$; e $\delta_{\mathrm{ijk}}$ : erro aleatório c.

Para a avaliação da adaptabilidade e estabilidade, utilizaram-se os métodos de Eberhart e Russel (EBERHART \& RUSSELL, 1966), e Lin e Binns (LIN \& BINNS, 1988).

O método de Eberhart e Russel baseia-se no seguinte modelo de regressão linear: $Y_{\mathrm{ij}}=\beta_{\mathrm{oi}}+\beta \mathrm{I}$ $+\delta_{i j}+\bar{\varepsilon}_{i j}$, em que $Y_{i j}$ é a média da cultivar i no ambienté $\mathrm{j} ; \beta_{\mathrm{oi}}$ eqüivale à média geral da cultivar $\mathrm{i} ; \beta_{1 \mathrm{i}}$ corresponde ao coeficiente de regressão linear, cuja estimativa representa a resposta da cultivar $i$ à variação do ambiente $\mathrm{j} ; \mathrm{I}_{\mathrm{j}}$ é o índice ambiental codificado; $\delta_{\mathrm{ij}}$ eqüivale aos desvios da regressão; e $\bar{\varepsilon}_{\mathrm{ij}}$ corresponde ao erro experimental médio.

As estimativas dos parâmetros de adaptabilidade e estabilidade são a média do genótipo $\left(\beta_{\mathrm{oi}}\right)$ e o coeficiente de regressão linear $\left(\beta_{1 \mathrm{i}}\right)$. De acordo com esta metodologia, a adaptabilidade é a capacidade das cultivares aproveitarem vantajosamente o estímulo do ambiente. São de adaptabilidade geral as cultivares com $\beta_{1 \mathrm{i}}=1$, adaptabilidade específica a ambientes favoráveis aquelas com $\beta_{1 \mathrm{i}}>1$ e adaptabilidade específica a ambientes desfavoráveis aquelas com $\beta_{1 \mathrm{i}}<1$. O parâmetro de estabilidade $\sigma_{\mathrm{di}}^{2}$ é estimado pelo método da análise de variância, a partir do quadrado médio do desvio da regressão de cada cultivar (QMDi) e do quadrado médio do resíduo (QMR), onde $\sigma^{2}{ }_{\text {di }}=(\mathrm{QMDi}-\mathrm{QMR}) / \mathrm{r}$. A estabilidade refere-se à previsibilidade da cultivar em relação ao modelo de regressão linear. São considerados estáveis as cultivares com desvios de regressão não-significativos e instáveis aquelas com desvios significativos.

O método de Lin e Binns estima a estatística $\mathrm{P}_{\mathrm{i}}$, que é a medida de adaptabilidade e estabilidade de comportamento em um único parâmetro. $\mathrm{O} \mathrm{P}_{\mathrm{i}}$ é estimado pela seguinte fórmula $P_{i}=\frac{\sum_{i=1}^{n}\left(X_{i j}-M_{j}\right)^{2}}{2 n}$, 
em que $\mathrm{P}_{\mathrm{i}}$ é a estimativa de adaptabilidade e estabilidade da cultivar $\mathrm{i}$; $\mathrm{X}_{\mathrm{ij}}$ é a produtividade da cultivar i no ambiente $\mathrm{j}$; $\mathrm{M}$ é a resposta máxima observada entre todas as cultivares no ambiente $\mathrm{j}$; $\mathrm{n}$ é o número de ambientes. Assim, o parâmetro $\mathrm{P}$ relaciona a distância da cultivar avaliada à cultivar que apresentou maior produtividade obtida em cada corte, de modo que quanto menor o seu valor, maior será a adaptabilidade e estabilidade de comportamento da cultivar em questão. As análises de estabilidade e adaptabilidade foram realizadas utilizando o programa GENES (CRUZ, 2001).

\section{RESULTADOS E DISCUSSÃO}

Não houve significância para o efeito de cultivares (Tabela 1), o que pode ter sido causado pelo elevado coeficiente de variação entre parcelas de cultivares. Entretanto, os efeitos de corte e da interação cultivares x cortes foram significativos. A significância da interação cultivares $x$ cortes indica que o comportamento relativo das cultivares não foi o mesmo em todos os cortes, ou seja, as cultivares mais produtivas num dado corte podem não ter apresentado desempenho superior numa outra avaliação. A identificação de cultivares que apresentem ampla adaptabilidade e alta estabilidade de produção de matéria seca, nos cortes realizados ao longo do ano, é de grande interesse. No caso da alfafa, que é muito utilizada em sistemas intensivos de produção de leite, a utilização de cultivares que apresentam maior oscilação de produção de forragem ao longo do ano, obrigam o produtor a utilizar maior quantidade de suplementação volumosa de outra fonte (silagem, feno,

Tabela 1 - Análise de variância para o caráter produção de matéria seca, avaliado em 35 cultivares de alfafa no período de 1996 a 1999. Sertãozinho, SP.

\begin{tabular}{lcc}
\hline Fontes de variação & GL & Quadrados médios \\
\hline Blocos & 2 & 320366,2 \\
Cultivar (Cv) & 34 & $640170,9 \mathrm{~ns}$ \\
Erro a & 68 & 437023,8 \\
Corte (Co) & 28 & $66844703,7 * *$ \\
Erro b & 56 & 570291,6 \\
Interação (Cv x Co) & 952 & $112898,6 * *$ \\
Erro c & 1904 & 74969,7 \\
CV a (\%) & 40,9 & \\
CV b (\%) & 46,7 & \\
CV c (\%) & 16,9 & \\
Média & 1616,7 & \\
\hline
\end{tabular}

ns Não-significativo. ${ }^{*}$ e ${ }^{* *}$ Significativos a 5 e $1 \%$ de probabilidade, respectivamente pelo teste $\mathrm{F}$. etc...) para manter a produção de leite por animal, o que acaba elevando o custo de produção de leite.

Pela metodologia de Eberhart e Russel, considerando que a cultivar selecionada será explorada tanto nos cortes realizados em condições ambientais favoráveis quanto nos desfavoráveis, não há interesse em selecionar cultivares que apresentem $\beta_{1 \mathrm{i}}>1$ ou $\beta<1$. A cultivar ideal será aquela que apresentar ${ }^{\mathrm{i}}$ alta produtividade, adaptabilidade geral $\left(\beta_{1 \mathrm{i}}=1\right)$ e boa previsibilidade $\left(\sigma_{\mathrm{di}}^{2}=0\right)$. Das 35 cultivares avaliadas, apenas 14 apresentaram adaptabilidade geral $(\beta=1)$ e boa previsibilidade $\left(\sigma_{\text {di }}^{2}=0\right)$. Dessas, apenals 10 apresentaram médias maiores que a média geral, que foram SW 8210, Victoria SP, MH 15, 5888, Araucana, Alto, Rio, BR 1, BR 3 e Maricopa (Tabela 2).

Com base nos resultados obtidos pelo método de Lin e Binns (Tabela 2), das 10 cultivares que apresentaram maior adaptabilidade e estabilidade (menores $\mathrm{P}_{\mathrm{i}}$ ), sete também apresentaram adaptabilidade geral e boa previsibilidade segundo a metodologia de Eberhart e Russell, que foram as cultivares SW 8210, Victoria SP, MH 15, 5888, Araucana, BR 1 e BR 3, todas com médias maiores que a média geral. Assim, ambas as metodologias apresentaram boa concordância em identificar as cultivares com maior adaptabilidade e estabilidade. Entretanto, houve discrepância na classificação da adaptabilidade e estabilidade da cultivar Monarca SP. Pelo método de Lin e Binns, essa cultivar foi a que apresentou melhor adaptabilidade e estabilidade para todos os cortes avaliados, com o menor valor de $\mathrm{P}_{\mathrm{i}}$, enquanto, pelo método de Eberhart e Russell, ela foi classificada como de comportamento melhor nos cortes realizados em condições ambientais favoráveis. Essa discordância se deve ao conceito de adaptabilidade das duas metodologias: o de Eberhart e Russell se baseia num coeficiente de regressão linear da produção da cultivar em função do índice ambiental, enquanto que o de Lin e Binns consiste numa comparação com a maior média em cada ambiente. De acordo com esta última metodologia, a cultivar cujas produtividades, em cada ambiente, estiverem mais próximas da máxima produtividade obtida em cada corte, obterá um $\mathrm{P}_{\mathrm{i}}$ de baixa magnitude, sendo considerado de alta estabilidade $\mathrm{e}$ adaptabilidade.

Os dois métodos de avaliação de adaptabilidade e estabilidade utilizados foram eficazes na identificação de cultivares de alfafa de ampla adaptabilidade e alta estabilidade de produção de matéria seca, podendo serem utilizados como uma técnica auxiliar na identificação de genótipos superiores de alfafa. As cultivares SW 
Tabela 2 - Estimativa dos parâmetros de adaptabilidade e estabilidade obtidos pelos métodos de Eberhart e Russel, e Lin e Binns, para o caráter produção de matéria seca.

\begin{tabular}{|c|c|c|c|c|}
\hline \multirow{2}{*}{ Cultivares } & \multicolumn{2}{|c|}{ Eberhart e Russel } & \multirow{2}{*}{ Média (kg/ha) } & \multirow{2}{*}{$\frac{\text { Lin e Binns }}{\mathrm{P}_{\mathrm{i}}}$} \\
\hline & $\beta_{1 \mathrm{i}}$ & $\sigma_{\mathrm{di}}^{2}$ & & \\
\hline Valley Plus & $0,96^{\mathrm{ns}}$ & $20.993,3 * *$ & $1.538,8$ & 154.071 \\
\hline WL516 & $0,85^{* *}$ & $8.715,8 \mathrm{~ns}$ & $1.544,9$ & 163.160 \\
\hline Alfa 200 & $0,90^{* *}$ & $0,0 \mathrm{~ns}$ & $1.644,4$ & 104.718 \\
\hline Falcon & $0,88^{* *}$ & $31.745,5 * *$ & $1.563,8$ & 161.529 \\
\hline SW 8210 & $1,02^{\mathrm{ns}}$ & $0,0 \mathrm{~ns}$ & $1.751,5$ & 54.027 \\
\hline SW 8112 A & $0,93^{\mathrm{ns}}$ & $0,0 \mathrm{~ns}$ & $1.597,1$ & 113.049 \\
\hline Alto & $0,98^{\mathrm{ns}}$ & $0,0 \mathrm{~ns}$ & $1.669,3$ & 89.349 \\
\hline Rio & $1,02^{\mathrm{ns}}$ & $0,0 \mathrm{~ns}$ & $1.665,3$ & 85.994 \\
\hline ICI 990 & $0,86^{* *}$ & $10.643,4 \mathrm{~ns}$ & $1.497,3$ & 179.567 \\
\hline Monarca SP & $1,09^{*}$ & $0,0 \mathrm{~ns}$ & $1.753,0$ & 44.168 \\
\hline Victoria SP & $1,05^{\mathrm{ns}}$ & $5.338,8 \mathrm{~ns}$ & $1.717,2$ & 70.647 \\
\hline Esmeralda SP & $0,98^{\mathrm{ns}}$ & $0,0 \mathrm{~ns}$ & $1.596,3$ & 103.374 \\
\hline Costera SP & $1,19^{* *}$ & $16.272,6 *$ & $1.488,9$ & 165.164 \\
\hline Semit 711 & $1,09^{*}$ & $2.484,8 \mathrm{~ns}$ & $1.567,9$ & 121.073 \\
\hline Semit 921 & $0,85^{* *}$ & $4.470,4 \mathrm{~ns}$ & $1.515,9$ & 174.596 \\
\hline Araucana & $1,07^{\mathrm{ns}}$ & $0,0 \mathrm{~ns}$ & $1.673,2$ & 66.763 \\
\hline Maricopa & $1,05^{\mathrm{ns}}$ & $0,0 \mathrm{~ns}$ & $1.631,5$ & 93.413 \\
\hline Sutter & $0,98^{\mathrm{ns}}$ & $18.541,7 *$ & $1.527,8$ & 158.253 \\
\hline P 30 & $1,08^{*}$ & $34.100,1 * *$ & $1.476,7$ & 181.830 \\
\hline P 205 & $1,18^{* *}$ & $36.432,0 * *$ & $1.422,2$ & 202.903 \\
\hline F 708 & $1,06^{\mathrm{ns}}$ & $5.963,3 \mathrm{~ns}$ & $1.614,0$ & 102.157 \\
\hline F 686 & $1,15^{* *}$ & $0,0 \mathrm{~ns}$ & $1.648,3$ & 83.790 \\
\hline El Grande & $1,01^{\mathrm{ns}}$ & $0,0 \mathrm{~ns}$ & $1.550,3$ & 124.653 \\
\hline 5929 & $0,88^{* *}$ & $4.832,7 \mathrm{~ns}$ & $1.587,9$ & 132.925 \\
\hline Florida 77 & $1,04^{\mathrm{ns}}$ & $24.237,8 * *$ & $1.691,5$ & 79.782 \\
\hline 5888 & $0,96^{\mathrm{ns}}$ & $0,0 \mathrm{~ns}$ & $1.714,6$ & 67.836 \\
\hline 5715 & $0,84^{* *}$ & $27.009,2 * *$ & $1.720,7$ & 98.400 \\
\hline MH 4 & $1,11^{* *}$ & $8.786,0 \mathrm{~ns}$ & $1.596,8$ & 115.344 \\
\hline MH 15 & $1,06^{\mathrm{ns}}$ & $11.383,6 \mathrm{~ns}$ & $1.715,7$ & 68.894 \\
\hline BR 1 & $0,99^{\mathrm{ns}}$ & $0,0 \mathrm{~ns}$ & $1.663,1$ & 82.114 \\
\hline BR 2 & $1,05^{\mathrm{ns}}$ & $12.618,4 *$ & $1.742,6$ & 59.768 \\
\hline BR 3 & $1,02^{\mathrm{ns}}$ & $0,0 \mathrm{~ns}$ & $1.660,2$ & 78.371 \\
\hline BR 4 & $0,92^{*}$ & $0,0 \mathrm{~ns}$ & $1.563,5$ & 130.708 \\
\hline SW $9210 \mathrm{~A}$ & $0,99^{\mathrm{ns}}$ & $17.486,9 *$ & $1.685,3$ & 85.610 \\
\hline Crioula & $0,91^{*}$ & $55.879,1 * *$ & $1.587,0$ & 159.244 \\
\hline Média & & & $1.616,7$ & \\
\hline
\end{tabular}

${ }^{\text {ns }}$ Não significativo. ${ }^{*} \mathrm{e}^{* *}$ Significativo em nível de 5 e $1 \%$, respectivamente, pelo teste t.

$\left(\right.$ Ho: $\left.\beta_{1 i}=1,0\right)$ e pelo teste $F\left(H o: \sigma_{\text {di }}^{2}=0\right)$.

8210, Victoria SP, MH 15, 5888, Araucana, BR 1 e BR 3 foram as de maior adaptabilidade e estabilidade de comportamento.

\section{REFERÊNCIAS BIBLIOGRÁFICAS}

CARVALHO, L.P. et al. Adaptabilidade e estabilidade em cultivares de algodão herbáceo. Pesquisa Agropecuária Brasileira, Brasília, v.30, n.2, p.207-213, 1995.
CRUZ, C.D.; REGAZZI, A.J. Modelos biométricos aplicados ao melhoramento genético. 2.ed. Viçosa : UFV, 1997. 390p.

CRUZ, C.D. Programa GENES: versão Windows; aplicativo computacional em genética e estatística. Viçosa : UFV, 2001. 648p.

EBERHART, S.A.; RUSSELL, W.A. Stability parameters for comparing varieties. Crop Science, Madison, v.6, p.36-40, 1966. 
LIN, C.S.; BINNS, M.R. A superiority measure of cultivar performance for cultivar $\mathrm{x}$ location data. Canadian Journal of Plant Science, Ottawa, v.68, p.193-198, 1988.

MONTEIRO, A.L.G.; CORSI, M.; CARVALHO, D.D. Freqüência de corte e intensidade de desfolha em duas cultivares de alfafa (Medicago sativa L.). I. Peso, número, produção estacional e dinâmica de aparecimento das brotações basilares. Revista Brasileira de Zootecnia, Viçosa, v.28, n.3, p.446452, 1999.

OLIVEIRA, J.S. et al. Adaptabilidade e estabilidade de cultivares de milho para silagem em relação à produção de matéria seca degradável no rúmen. Revista Brasileira de Zootecnia, Viçosa, v.28, n.2, p.230-234, 1999.

RAMALHO, M.A.P.; FERREIRA, D.F.; OLIVEIRA, A.C. de. Experimentação em genética e melhoramento de plantas. Lavras : UFLA, 2000. 326p.

SILVA, F.G.; ANUNCIAÇÃO FILHO, C.J.; TABOSA, J.N. Estabilidade da produção de grãos de arroz irrigado nos Estados de Alagoas e de Pernambuco. Pesquisa Agropecuária Brasileira, Brasília, v.30, n.3, p.347-351, 1995.

VILELA, D. Potencialidade da alfafa na Região Sudeste do Brasil. I nforme Agropecuário, Belo Horizonte, v.16, n.175, p.50-53, 1992. 